\title{
Efficacy of traditional Chinese herbal medicine in the treatment of gastrointestinal polyps and chronic gastritis: A case report
}

\author{
Xiaoqiong Luo ${ }^{1,2}$, Wei Jin ${ }^{2}$, Qingjie $\mathrm{Li}^{3}$, Yang Song ${ }^{1,2}$ and Xiaoyun Zhang ${ }^{1,2}$ \\ ${ }^{1}$ Clinical Medicine College, Chengdu University of Traditional Chinese Medicine, Chengdu, Sichuan 610072, ${ }^{2}$ Emergency \\ Department, ${ }^{3}$ Department of Vascular Surgery, The Teaching Hospital of Chengdu University of Traditional Chinese Medicine, \\ Chengdu, Sichuan 610075, China
}

*For correspondence: Email: zhangxy1980@126.com

\begin{abstract}
Background: The rate of gastrointestinal adenomatous polyps, often regarded as precancerous lesions, developing into cancer is $40-70 \%$. Endoscopic resection has been the preferred method for treating gastric polyps since the late 1960s. Surgical removal of polyps continues to play an important role in the treatment of polyps; however, the efficacy of such treatment cannot be guaranteed, and polyps may recur.

Case presentation: Here, we report a 44-year-old man suffering from gastrointestinal polyps who refused surgical treatment and instead was treated with traditional Chinese herbal medicine (TCHM) for approximately 1 year. The patient was diagnosed with "qi deficiency and dampness syndrome" based on traditional Chinese medicine theory and was treated with the TCHM "strengthen qi and remove dampness formula", referred to as the shen-ling-bai-zhu powder/decoction.

Conclusion: This case suggests that TCHM may play an important role in the treatment of gastrointestinal polyps. Relevant data are, however, limited and a randomized controlled trial is needed to confirm TCHM efficacy in a larger population.
\end{abstract}

Keywords: Case study, Gastrointestinal polyps, Traditional Chinese herbal medicine, Shen-ling-bai-zhu powder

\begin{abstract}
Tropical Journal of Pharmaceutical Research is indexed by Science Citation Index (SciSearch), Scopus, International Pharmaceutical Abstract, Chemical Abstracts, Embase, Index Copernicus, EBSCO, African Index Medicus, JournalSeek, Journal Citation Reports/Science Edition, Directory of Open Access Journals (DOAJ), African Journal Online, Bioline International, Open-J-Gate and Pharmacy Abstracts
\end{abstract}

\section{INTRODUCTION}

Gastric polyps constitute a common lesion of the gastric mucosal epithelium. These lesions can be divided into adenomatous polyps, hamartoma polyps, inflammatory polyps, and hyperplastic polyps according to their histological characteristics [1]. Gastric polyps are often regarded as precancerous lesions, and the rate of adenomatous polyp development into cancer is $40-70 \%$ [2].Gastric polyps develop as a result of chronic gastritis, Helicobacter pylori $(\mathrm{Hp})$ infection, bile reflux of the stomach following by-pass surgery as well as genetic and environmental factors [3].

Many clinical manifestations of polyps such as abdominal discomfort, nausea, vomiting, and gastrointestinal bleeding (due to mucosal erosion or ulcers), can only be observed when complications occur [4]. Since the late 1960s, gastric polyps have been resected endoscopically. This has become the preferred method of treatment. Surgical removal of polyps plays an important role in the treatment of polyps 
[2,4]; however, its efficacy cannot be guaranteed. Moreover the polyps can recur.

In recent years, traditional Chinese herbal medicine (TCHM) has gradually been demonstrated to be effective for the symptoms of gastric diseases, such as functional dyspepsia $[5,6]$ and diarrhea $[7,8]$. Data pertaining to TCHM treatment of gastrointestinal polyposis are however rare. Here, we report the case of a patient who suffered from gastric polyps and was treated for approximately one year by the administration of shen-ling-bai-zhu (SLBZ) powder/decoction.

\section{CASE PRESENTATION}

A 44-year-old man presented at the Chengdu University of Traditional Chinese Medicine Teaching Hospital on the 3rd of April, 2013 with a one year, history of weakness and associated loss of weight. There was an exacerbation of symptoms two weeks prior to presentation. He was feeble and weak with no obvious predisposing factor. He admitted to having lost $10 \mathrm{~kg}$ body weight during the past year. There was associated dizziness, flustering, hunger, and hot flashes. There was no history of cough, hemoptysis, insomnia, urinary frequency or urgency, dysuria. His past medical history included chronic hepatitis lasting more than 10 years, a rectal polyp resection 1 year previously, and 6 months of chronic gastritis. The patient was a technician employed by a pilot group in Chengdu city, Sichuan Province, and had not come in contact with dogs, cats, or birds. He had not traveled outside the city for a long time. There was no history of recent sexual contacts or recreational drug use. He was an occasional alcohol drinker and never smoked.

Nothing remarkable was detected on a physical examination. His oxygen saturation was $98 \%$ on ambient air, heart rate was 92 beats per minute, blood pressure was 123/81 $\mathrm{mmHg}$, blood glucose level was $6.3 \mathrm{mmol} / \mathrm{l} 4$ hours after eating, and respiratory rate was 19 breaths per minute.

A chest radiograph showed no abnormalities. However, gastroscopy revealed two $2-4 \mathrm{~mm}$ polypoid bodies near the pylorus, duodenal bulb and at the greater curvature, respectively (Figure 1). Histological examination of the polypoid bodies showed chronic inflammation of the mucous membranes of the polyps in the duodenal bulb area. The ${ }^{14} \mathrm{C}$-urea breath test was positive for $\mathrm{Hp}$. The endoscopic findings of chronic atrophic gastritis and multiple duodenal bulb polyps respectively are shown in Figure 1.

\section{Syndrome differentiation}

The patient refused a gastrointestinal polypectomy and rather asked for TCHM therapy. According to the traditional Chinese medicine (TCM) principle of the five zang organs, one of the main physiological functions of the spleen is to govern transportation and transformation, such as digestion, absorption, and transmission of nutrients. The function of the spleen is dependent on the condition of spleen qi. Digestion, absorption, and transmission are normal when spleen qi is abundant. A deficiency in spleen qi may lead to symptoms, such as anorexia, abdominal distention, diarrhoea, lassitude, loss of weight, and malnutrition. Moreover, a serious deficiency of spleen qi may result in retention of water and fluid, as both transportation and transformation are decreased.

Retention of water and fluid often manifests as phlegmatic hygrosis condensation and gradually develops into polyps or nodulus in the body (namely accumulation of dampness due to spleen qi deficiency). This manifest vocal nodules, intestinal polyps, and nasal polyps.

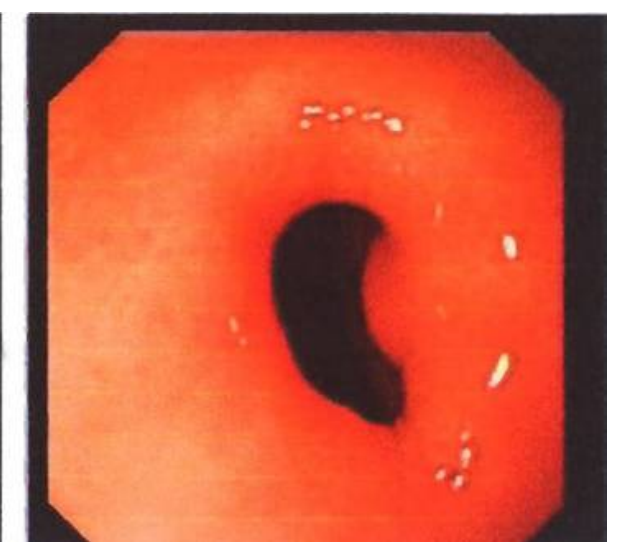

Figure 1: Initial endoscopic results. (a) Duodenal bulband (b) polyp in the pyloric area. 
The patient presented with a pale and/or white and thick greasy coating on his tongue with teeth marks on both sides of the tongue In addition, there was a soggy or slippery pulse as a result of qi deficiency and dampness syndrome.

SLBZ powder/decoction (SLBZP) is a TCHM formula that strengthens spleen qi and removes dampness in line with the TCM monograph taiping huimin and agent pharmacopoeia discovered during the Song dynasty of ancient China. This formula has been widely used to protect intestinal mucosal barrier function, improve chronic diseases, such as dampness accumulation due to spleen qi deficiency syndrome, and restore gastrointestinal dysfunction [9]. In addition, SLBZP was effective as an adjuvant therapy in a chemotherapyinduced animal model of hepatocellular carcinoma, it may act by inhibiting tumor growth inducers [10]. The apparent therapeutic principle of SLBZP is to strengthen qi and remove dampness.

The patient had a reddish tongue covered with a thin-white coating and a smooth pulse. We administered SLBZP after confirming the syndrome based on TCM theory. The SLBZP was taken at a dose of $100 \mathrm{ml}$ three times per day. According to TCM theory, raw or cold foods, oily food and fishy foods are forbidden during treatment. In addition, some difficult-todigest foods and other stimulating foods should be avoided while taking any kind of TCM. The composition of the SLBZP is shown in Table 1. All herbs was purchased from Si Chuan Neautus Traditional Chinese Medicine, Inc (Sichuan, China).

\section{RESULTS}

After 5 days of consuming the SLBZP, the patient's symptoms of palpitations, tiredness, and weakness improved significantly, with no adverse drug reaction recorded except for sore throat, morning nausea accompanied by vomiting of clear water, and anorexia.

After 8 months of intermittent use of the SLBZP lasting till December 14, 2013, the patient informed us that his nausea and vomiting had disappeared and the associated weakness had reduced. Repeat gastroscopy showed several small 2-4 mm smooth polyps scattered near the pyloric duodenal bulb. Because the ${ }^{14} \mathrm{C}$-urea breath test was negative for $\mathrm{Hp}$, we advised the patient to continue taking the SLBZP.

At the third follow-up in June11, 2014, the weakness had subsided. Gastroscopy revealed neither ulcer, folded nodules nor polyps in the stomach. The gastric mucosa appeared and displayed the usual normal red and white color (Figure 2). The polyp in duodenal bulb and pyloric area had disappeared. No negative symptom was reported.

\section{DISCUSSION}

The patient was diagnosed with "qi deficiency and dampness syndrome" based on TCM theory and was treated with the TCHM "strengthen qi and remove dampness formula". The gastrointestinal polyps in this patient were treated successfully, and the associated symptoms disappeared without complications during the follow-up.

Table 1: Composition of the Traditional Chinese Herbal Medicine shen-ling-bai-zhu powder/decoction

\begin{tabular}{llll}
\hline Chinese name & Common name & Botanical name & $\%$ \\
\hline Ren shen & Ginseng & Radix Ginseng & 8.7 \\
Fu ling & Poria cocos & Poria & 4.3 \\
Chao Bai zhu & Rhizoma Atractylodis Macrocephalae & (Rhizoma Atractylodis & 8.7 \\
& & Macrocephalae) (baked) & 8.7 \\
Bai bian dou & Whitehyacinth Bean & Semen Dolichoris Album & 4.3 \\
Chen pi & Pericarpium citri reticulatae & Pericarpium Citri Reticulatae & 4.3 \\
Sha ren & Fructus amomi & Fructus Amomi & 8.7 \\
Shan yao & Chinese yam & Rhizoma Dioscoreae & 8.7 \\
Yi ren & Semen Coicis & Coicis semen & 4.3 \\
Jie geng & Platycodon grandiflorum & Radix Platycodi & 8.7 \\
Gan jiang & Rhizoma zingiberis & Rhizoma Zingiberis & 4.3 \\
Shen qu & Chinese drug Shenqu & Massa Dermentata Medicinalis & 8.7 \\
Shan zha & Fructus crataegi & Fructus Crataegi & 4.3 \\
Ban xia & Pinellia ternata & Rhizoma Pinelliae & 4.3 \\
Jin ying zi & Fructus Rosae Laevigatae & Rosa Laevigata Michx & 4.3 \\
Sang piao xiao & Mantis Egg-case & Ootheca Mantidis & 4.3 \\
Zhi Gan cao & Glycyrrhiza & Radix Glycyrrhizae praeparatae \\
\hline
\end{tabular}




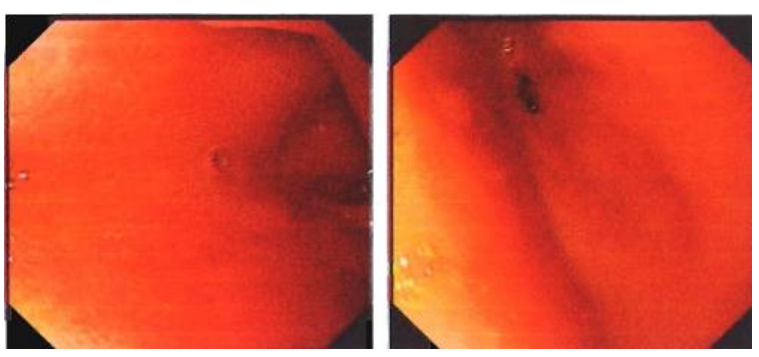

Figure 2: The endoscopic results in the patient after taking traditional Chinese medicine for approximately 14 months

Although limited data was obtained regarding the drug formula, its various components have been reported and confirmed by pharmacological research [10].

The main drug components of the formula boost the body's immune system and promote restoration from the diseased state. The Radix Ginseng, Poria, baked Rhizoma Atractylodis Macrocephalae, Semen Dolichoris Album, and Coicis semen present in the SLBZP enhance immunity [11]. Baked Rhizoma Atractylodis Macrocephalae protects the gastric mucosa and enhances intestinal motility [12]. Poria and Coicis semen have anti-inflammatory and anti-tumor effects. Coicis semen promotes $\mathrm{T}$ lymphocyte proliferation [13]. Semen Dolichoris Album enhances $T$ lymphocyte activity and has antibacterial and antiviral activities [14]. Radix Glycyrrhizae praeparatae protects the gastric mucosa. Nevertheless, no direct experimental evidence is available at present to explain the efficacy of the SLBZ ingredients on stomach and intestinal polyps. We advocate drug trials to identify the potential mechanism of the SLBZ and more rigorous randomized controlled trials to confirm its clinical effects on gastrointestinal polyps in the future.

\section{CONCLUSION}

We present a case report of gastrointestinal polyps treated with TCHM. The patient was diagnosed with "qi deficiency and dampness syndrome" based on TCM theory and was treated with the TCHM "strengthen qi and remove dampness formula" SLBZP. The gastrointestinal polyps and associated symptoms disappeared after approximately 1 year of TCHM therapy without any complications during the follow-up. This case suggests that TCHM could play an important role in the treatment of gastrointestinal polyps. It may be a better choice for the patients who refuse surgery or cannot be surgically operated on, because the Chinese traditional medicine treatment of chronic gastritis and gastrointestinal polyps method is simple and less painful. Relevant data are however limited, and randomized controlled trials are still needed to confirm its efficacy in a larger population.

\section{DECLARATIONS}

\section{Acknowledgement}

This study was supported by the Foundation of Research on Technology and Quality Standards of Mixture Formula for Regulating Stomach and Releasing Excessive Turbid (no. ZRQN1509, 030029031 to Wei Jin).

\section{Conflict of Interest}

No conflict of interest associated with this work.

\section{Contribution of Authors}

The authors declare that this work was done by the authors named in this article and all liabilities pertaining to claims relating to the content of this article will be borne by them.

\section{Open Access}

This is an Open Access article that uses a funding model which does not charge readers or their institutions for access and distributed under the terms of the Creative Commons Attribution License (http://creativecommons.org/licenses/by/ 4.0) and the Budapest Open Access Initiative (http://www.budapestopenaccessinitiative.org/rea d), which permit unrestricted use, distribution, and reproduction in any medium, provided the original work is properly credited.

\section{REFERENCES}

1. Park DY, Lauwers GY. Gastric polyps: classification and management. Arch Pathol Lab Med 2008; 132: 633-640.

2. Kim YH, Kim NG, Lim JG, Park C, Kim H. Chromosomal Alterations in Paired Gastric Adenomas and Carcinomas. Am J Pathol 2001; 158: 655-662.

3. Carmack SW, Genta RM, Schuler CM, Saboorian MH. The current spectrum of gastric polyps: a 1-year national study of over 120,000 patients. Am J Gastroenterol 2009; 104: 1524-1532.

4. Goddard AF, Badreldin R, Pritchard DM, Walker MM, Warren $B$. The management of gastric polyps. Gut 2010; 59: 1270-1276.

5. Qin F, Huang $X$, Ren $P$. Chinese herbal medicine modified xiaoyao san for functional dyspepsia: Meta-analysis of randomized controlled trials. $J$ Gastroenterol Hepatol 2009; 24: 1320-1325.

Trop J Pharm Res, November 2017; 16(11): 2780 
6. Xiao Y, Liu Yy, Yu Kq, Ouyang Mz, Luo R, Zhao Xs. Chinese herbal medicine liu jun zi tang and xiang sha liu jun zi tang for functional dyspepsia: meta-analysis of randomized controlled trials. Evid Based Complement Alternat Med 2012; 2012.

7. Singhuber J, Zhu M, Prinz S, Kopp B. Aconitum in traditional Chinese medicine: $A$ valuable drug or an unpredictable risk? J Ethnopharmacol 2009; 126: 18-30.

8. Gu Y, Zhang Y, Shi X, Li X, Hong J, Chen J, Gu W, Lu X, $X u$ G, Ning $G$. Effect of traditional Chinese medicine berberine on type 2 diabetes based on comprehensive metabonomics. Talanta 2010; 81: 766-772.

9. Tian L. Shen-ling-bai-zhu decoction scattered flavored rhubarb protect the intestinal mucosal barrier function of experimental research. Med J Liaoning 2011; 01: 9-11.
10. Xi S, Peng Y, Minuk GY, Shi M, Fu B, Yang J, Li Q, Gong $Y$, Yue $L, L i L$ et al. The combination effects of ShenLing-Bai-Zhu on promoting apoptosis of transplanted H22 hepatocellular carcinoma in mice receiving chemotherapy. J Ethnopharmacol 2016; 190: 1-12.

11. Jia Taiyuan BHS. Dangshen for $J 774$ rat effect of the activity of macrophage cell. Lishizhen Med Mater Med Res 2010; 11: 769-770.

12. Wangruina. The pharmacological effects and the comprehensive development and utilization of medicinal atractylodes. Anhui Ag Sci 2010; 38: 5610-5611, 5627.

13. Wu Y, Yuan Y. Advance in studies on chemical components and pharmacological effect of Semen Coicis. West Pharm J 2010; 25: 111-113.

14. Lu J, Cai J, Dai Y. Research progress of Semen Dolichoris Album. Hubei J TCM 2013; 35: 77-79. 